\title{
DISCUSSION.
}

\section{GENETIC MODES AND THE MEANING OF THE PSYCHIC. ${ }^{1}$}

When we can explain chemical affinity we may attempt to explain instinct; when we have explained instinct we may attempt intelligence. The explanation offered by dynamic realism' of the 'meaning' of the simplest of natural phenomena will presumptively be the explanation of the principle underlying all reactions.

We may ask why a comet pursues a given course rather than another. The answer is two-fold. First, because of the nature of the forces constituting the comet; second, by reason of the combinations of energy existing in the universe through which it passes. In other words, the trajectory of the comet is determined by correspondences existing between the comet and its environment. We might say that the trajectory of the comet is its path of least resistance, but this is only part of the truth. The nature of the energic structure of the comet is also a factor - the most important one. It has, we say, a certain mass of gravity. It has that which makes it a positive energic element in a universe of energy. It might be considered fanciful to suppose that as the extrinsic pull which draws the mother to her child has also its intrinsic side called affection, so there is an intrinsic affection corresponding to the extrinsic pull of the planet. Nevertheless, all analogy would indicate that, if not an affection or instinct, there is nevertheless an intrinsic element in all these cases.

So with the chemical element, all that we know about it consists in reactions, $i$.e., interferences of some type of energy with the energic complex of the environment. One of the most important of these reactions is what we call chemical affinity. If we indicate the locus formula of sodium by $\mathrm{Na}$ and that of chlorine by $\mathrm{Cl}$, then the expression $\mathrm{NaCl}$ (common salt) means that these two loci have certain

${ }^{1}$ A fragment found among the author's papers and submitted by $\mathrm{C}$. J. Herrick. - ED.

"Some of the implications of this term as used by the author will be found in his later writings, particularly, 'Fundamental Concepts and Methodology of Dynamic Realism,'Jour. Phil., Psych., Sci. Methods, Vol. I, No. II, 1904; and 'The Law of Congruousneas and its Logical Application to Dynamic Realism,' Ibid., Vol. I, No. 22, 1904.-C. J. H. 
compatibilities or correspondences which result, the energic complex being what it happens now to be in this particular environment, in a closer articulation or assimilation in these particular loci than between the activities expressed by $\mathrm{Na}_{2}$ and $\mathrm{H}_{3} \mathrm{O}$, for example. Under other conditions of environment, say at a high temperature or in the presence of larger amounts of water, the chemical affinity, as this harmony is called, would not be apparent. Now the cube of salt deposited from saturated solution is an expression to eye and touch of a mose or less permanent association of the types of energy labelled $\mathrm{Na}$ and $\mathrm{Cl}$ respectively. It is not true that $\mathrm{Na}$ and $\mathrm{Cl}$ are present in salt; they are potentially present in the sense that under certain conditions these two loci emerge from the complex with the same value they possessed when they entered it. $\mathrm{NaCl}$ is a new energy complex capable of reacting in its own appropriate way (dependent upon its own genetic mode) and is different from either $\mathrm{Na}$ or $\mathrm{Cl}$. It is not an algebraic sum of the energies $\mathrm{Na}$ and $\mathrm{Cl}$, but a trajectory resulting from their blending. Salt occupies a definite position in nature and is capable of impressing its energy upon other energic units in a way peculiarly its own. Thus, no other substance tastes as salt does. Now if there be an intrinsic side of the activity, $\mathrm{NaCl}$, that too may be totally unlike that of any other chemical substance. We say salt has an affinity for water. Does it thirst? When the human organism is dehydrated by evaporation due to exercise or the injestion of water imbibing substances, the state of receptivity to water or disturbed equilibrium existing in the tissues of the body is converted into a special nervous affection which may even become an element in consciousness and build up the most elaborate system of associations. But at some early point in this process we may discover simply living tissue needing water and back of this certain chemical substances with an affinity for water - in other words, exactly the same thing that $\mathrm{NaCl}$ has.

This disposition to change its form by uniting with another element is illustrated by the formation of all solutions and it is a mistake to suppose that a substance in solution is the same as a substance in solid form. It has claims to be called the same substance only because it can be evaporated out. But in the course of this process there is always a complete change of properties. Solid salt is not salty to the taste, salt in solution is not cubical. In short, we must school ourselves to see in the so-called elements or substances energic complexes whose form (nature) is at once determined by their primary locus formula and the impact or effect of the environment. So true is this that any substance can be fully understood only by knowing its pri- 
mary form and also the totality of its reaction with the environment. This is perhaps quite unlike our naive apprehension of objects which seem to have complete objective independence. The simplest experiment illustrates the error, however. We suddenly remove the support beneath the vase and instead of a thing-of-beauty in repose, we have a thing-in-notion and then a thing-in-a-hundred-pieces. The vase is just as really dependent, so far as being what it seems is concerned, on connections with the environment as the flower is which withers when removed from the parent stem.

Now the existence of any typical form of energy, say a crystal, in any energic complex is a fact of interaction. If a broken crystal is plunged into a suitable medium, it will be restored (this process goes on in rocks in case of metamorphism). The presence of the crystal acts as a determinant for the aggregation of other masses. The extraneous energy associates itself with the preëxisting types in accordance with the types of energy already called into being. The most noted instance of this power is in the case of animate matter. The most astounding fact in nature is perhaps the power of a worm or a man to ingest the same materials and create in one case worm substance and in the other human tissue. In the case of the crystal there may be millions of microliths contained in one crystal and all are alike or similar. In the case of the man there are millions of cells and we are able to distinguish groups of coordinated types.

The harmony between a particular energic type and its environment may be relatively stable or it may be dependent upon a high degree of constancy or invariability in that environment. Again, the energic unit may be progressively alternating or cyclical. Such a condition is found in the individual life which, like the trajectory of a planet, passes through a variety of progressively adjusted relations to the environment or comes into relation progressively to different environments. That type of energic unit which passes consecutively into relations with different energic complexes will alter its locus formula. When water passes into a gaseous state it is no longer water.

Here is a moving point. I, as a geometer, make 'cross-sections' of that point in relation to its environment and construct a locus (say $\left.y^{2}-2 p x\right)$. But in doing all this I have not produced the concept of a parabola such as I get when I see one. I go on varying the locus formula and produce successively a circle, an ellipse, etc. You may say that these things can be predicted in advance. The series of locus formulæ might be, but no power would enable us to experience a 
circle till I saw it. Each new form has a meaning (differentia) in experience peculiar to it.

Now, as a biologist, I have no doubt that the various sense organs arose by successive variations from some primitive type. As dynamic monist (or functional psychologist, if you prefer), I consider the psychical and physical to be two ways of expressing a real activity. But, as 'psychic' (Baldwin's limitation '), my subjective experience is very different when visual and tactual sensations respectively are evoked. As has so often been said, there is no reason why certain vibrations awaken sensations of green and others of sweet.

When eyes came in vogue, a new thing, a new 'genetic mode' arose. You could never have predicted it. You might have predicted the size and form of the rods and cones and the index of refraction of the lens but the subjective interpretation in intimate experience is not a priori predictable. It is conceivable that a child might, by unconscious movements, happen upon a sensation entirely new to it. The series of 'psychic' events is not subject to scientific analysis. The subsequent psychological construction is wholly synthetic and consists in relational redistribution and combination. These may be construed among themselves and with other facts which we do not call psychological.

It may be said that the modes of immediate consciousness are the only ones that could be genetic in this sense, that all others could be predicted from the earlier. These are doubtless the only ones we can know anything about. The power of prediction rests upon the presumption of the cyclical nature of action- 'uniformities' we call these cycles, whether heart-beats or eclipses. If we project these cycles on a 'cross-section' of experience, our predictions are valid in that plane. We may have as complete a system as possible plotted by our science, like a plot of hundreds of observations upon some movable star and may, on this basis, lay out the orbit fully upon the plane of experience, but this is not the same thing as the star moving in space. The 'meaning' of this we could perhaps only discover by being the star. All this may be another name for the limitation of knowledge, but it is a necessary limitation of knowledge and has to be reckoned with. But if a thing be truly genetic, every new stage is really new and not a repetition, nor can we know from the past what new value may attach to the progressive modifications. We must let zo of the cause-and-effect traditions - never backward turn the wheels of time.

' Baldwin's Dict. of Philos., art. 'Psychic or Mental.' 
A corollary is that another stage of being is ' genetically' possible in which the energy of the present shall be elaborated in such forms as may present to experience something totally inconceivable to the heart of man.'

The further question arises, however, (and this is not so easy to answer) if genetic (psychic)' modes arise that have no predicaments in the past and no necessary determinants in the present, how do they cohere in a universe - how belong in an organic whole? The answer is, "They do not."

The 'psychic,' as psychic, is neither parallel to anything nor set in any kind of serial, or other, nexus with anything else. Anything possessing such relations would necessarily be predictable, $i$.e., to a being having complete knowledge. But no being can know what I feel. All the generalizations I make regarding the data furnished in immediate consciousness (everything psychological, in other words) I may relate or communicate, the peculiar tone or flavor of consciousness (its meaning) can neither be imparted nor anticipated. When we develop an organ for the ultra-violet rays we shall experience a new 'genetic mode,' but if the anticipations of science go far enough, we may not thereby get a single new psychological element; we shall simply find a value for this particular $x$ and all is in.

This sphere of epiphenomena can only be interpreted by reference to the metaphysical predicate of individuality. The three necessary forms or categories of our thinking, time, space and mode, each contributes to the definiteness of experience by conditioning it. Mode is that condition which is indispensable to individuality. Time is the necessary form of inner experience, space of outer experience, mode is prerequisite to all experience - it is the form of all experience.

We do not expect to encounter space or time 'spatzierend' by themselves. We do not try to line them up with the contents of special experiences and to make them cohere in a system with these. No more can we take the predicament of individuality in experience and set it in relations. I can say a great deal about green things. I can predict that they will arrive in April in special forms, but that which makes greenness different from sweetness or $b$ flat belongs to the formal category of mode. We have a sense of spatial extension, of temporal limitation, and, in like manner of special peculiarity. This is the tag which gives rise to the sense of other-ness or difference.

'It does not appear that Professor Baldwin limits genetic modes to the psychic, but I am of the opinion that it is safe to use that term only within these limits, if non-predicableness is insisted on in their definition. 
So far as we are concerned, then, the genetic modes find their illustrations in the psychic - in our own peculiar content of experience, but it may be that every form of self-centered experience - all forms of vector activity, at least - have their inner meaning and that this, again, is reflected upon the great centre of reference of the whole system as a total meaning. This form of self-interpretation of energy that we call consciousness may be one of an innumerable multitude of similar incommunicable experiences which taken together form the real 'meaning' of the world.

FORMERLY OF DENISON UNIVERSTYY.

C. L. HerRick. ${ }^{1}$

1 Deceased. 